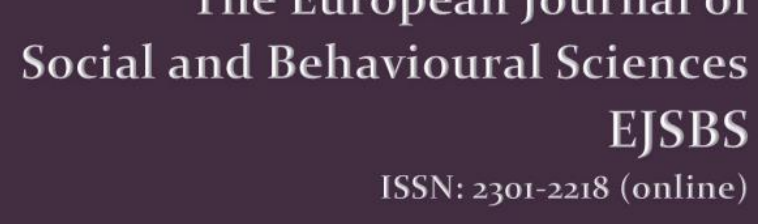

The European Journal of Social and Behavioural Sciences

EJSBS Volume V, Issue II (e-ISSN: 2301-2218)

\title{
THE EMPOWERMENT MODEL OF PARENT CONSULTATION WITH THE POSITIVE PSYCHOLOGY PERSPECTIVE
}

\author{
Payling Harn ${ }^{\mathrm{a} *}$, Peili Wu \\ ${ }^{a}$ University of Hsuan Chuang, Assiatnat Professor of Appied Psychology, Hsuan Chuang Rd., no.48, Hsinchu, \\ Taiwan \\ ${ }^{b}$ National Taiwan Normal University, Professor of Educational Psychology and Counselling Helping, East Rd., \\ No:162, Tapei, Taiwan
}

\begin{abstract}
The aim of the study is to develop an indigenous parent consultation model based on the viewpoint of positive psychology, and to further discuss the empowerment development and transformation contexts of consultees in the process of consultation. Three data were collected from four female parents, which are the transcripts in the process of consultation, the transcripts of tracking interviews and the texts of tracking questionnaires, then analyzed the data by the temp late analysis method, to revise the initial version of parents empowerment concept model established by Gibson (1995). The results of the study are: (1) After giving cultural and behavioural connotation, the researcher further developed six empowerment subjects which are "Exploring and Tracing Back to the Origin", "Initiating Fortune from the Extreme Adversity", "Comprehending from Converting Thoughts", "Facilitating from Being Proficient", "Flourishing Strengths" and "Diversifying Crises into Safeties". (2) Empowerment transformation uses "Exploring and Tracing Back to the Origin" and "Initiating Fortune from the Extreme Adversity" as the principal line, then, to form a dynamic force which will transform the origin exploration to bittersweet. (3) The results of empowerment are to establish parenting wellbeing by the extensibility of relationship culturing, to promote the appropriate expression of parenting by awareness transformation, so as to create a power of "Diversifying Crises into Safeties".
\end{abstract}

Keywords: Positive psychology, parent cousultation, empowermnet model, template analysis method

(C) 2013 Published by C-crcs. Peer-review under responsibility of Editor(s) or Guest Editor(s) of the EJSBS.

${ }^{*}$ Corresponding author.

E-mail address: plharn@hcu.edu.tw

doi: $10.15405 /$ ejsbs. 65

This work is licensed under a Creative Commons Attribution-NonCommercial-NoDerivatives 4.0 International License. 


\section{Introduction}

Intensive efforts have been made by the educational and medical organizations to assist the development of mental adaptation among juveniles in recent years. The severe psychological troubles faced by the juveniles may both influence them physically and mentally, and even form the imp act on the family (Goldenberg \& Goldenberg, 2008). Although the related organizations had taken great efforts to assist the physical and mental adaptation of the juveniles, they seem to ignore the psychological care of their targets. Therefore, the importance of consultation began to be emphasized in the mental hygiene in Taiwan. If the professional knowledge, skill and objectivity of the people important to the target can be improved by professional consultation service, it will assist the target to develop positively, and an effective cooperating platform will be created to help the target more properly and more professionally (Lin, 2002; 2007; Qiu \& Wu, 2004; Wang, 2002; Wu, 1998).

In recent years, the positive psychology has led a view of increasing the wellbeing and efficacy of the target in the mental adaptation through enhancing the positive experience, which is different from the traditional medical models (Snyder \& Lopez, 2002). In addition, the positive psychology has been widely used in various mental hygiene practices. Qiu and $\mathrm{Wu}$ (2004) highlighted the positive communication between parents and children in their study on parent-child communication consultation group. The concept of parent empowerment began to be introduced in the parent support strategies. Parent empowerment refers to a social experience that parents identify and improve their ability to realize their demands, solve their problems and control their life by necessary resources (Gibson, 1995). By introducing the concept of empowerment, foreign scholar Gibson (1995) proposed five empowerment topics in the study on the maternal empowerment development in the family with children suffering chronic disease, namely, finding the reality, considering setback, criticizing and introspecting, controlling and maintaining. The "maintaining" means mothers can perceive their advantages and abilities, and show their control power over the dynamic environment, so that they can keep a regular self-control to meet the future challenges and difficulties. The parent consultation with the positive psychology perspective focuses on whether parents can gain full nourishing and empowerment to improve their wellbeing and significance in the parenting process.

As mentioned above, the study attempts to improve the positive adaptation process in parent consultation from the perspective of the positive psychology. However, it's difficult to ignore the social and cultural influence if the consultation is expected to be more adaptable to the parenting context of the consultees (Chin, Liem, Ham, \& Hong, 1993; Holcomb-McCoy $\&$ Bryan, 2010; Gibbs, 1990). The practical workers in Taiwan usually adopt the positively 
subjective experience, positive characters and positive systems developed in western cultures to implement the positive psychology (Guo, 2011). However, the points above should be adjusted if applied in the Chinese culture, which requires the practical workers to consider further.

Based on the study motivation mentioned above, the study combines the positive psychology with the Chinese parent-child views and the connotation of Chinese wellbeing construction in the parent consultation, to help the consultant adopt the view of positive reconstruction to form the advantageous ability of the parents, extend their solutions on the stressful family matters, improve their resilience under pressure and help their children in the psychological rehabilitation and adaptation. Therefore, the researcher establishes the parent consultation model based on the view of Chinese positive psychology and explores the empowerment experience and transformation process of the consultees.

\section{Methodology of the study}

\subsection{Study Objects (Consultees)}

The objects of the study are the parents whose children suffer from mental diseases or have significant behaviors that affect their life and study adaptation. The study conducted parent consultation during 2010.4 and 2011.4, and chose four consultees for data analysis of formal research. At the beginning, the study expected to cover the parenting roles of different genders. During the object selection, however, mothers have the higher intention of participating in the study. Therefore, the objects in the study are all females aging from 40 to 55. Two children of them are junior high school students and the other two are senior high school students; three children are female and the other is male. The main topics consulted by the four objects are psychological disease care, assistance on study adaptation, physical and mental adaptation of bully events, and the assistance on school refusal, all of which are the common physical and mental problems among the juveniles in campus. As for the consultation expectation, all the consultees want to improve the parent-child relationship and develop the physical and mental adaptation of children.

\subsection{Study Procedure}

The researcher served as consultant, data analyst and interviewer. As a consultant first, the researcher provided proper consultation frequency according to the characters and demands of the consultees. The consultation frequency is $5 \sim 7$ times. Besides, the sixth consultation for one of the consultees was conducted by two consultants together. 
After consultation, tracking interviews and questionnaire survey were conducted with the consultees to see the effectiveness and growth of their subjective perception. Moreover, the researcher analyzed the data with experts who have the professional background and practice experience on parent consultation after the consultation of the four consultees, to enhance the reliability and validity of the data analysis. The two analysts reach an agreement if the reliability of their consistency reaches .93 .

\subsection{Data Analysis}

After consultation, the consultees' response during consultation and the post interview content were taken as analysis text and analyzed by the temp late analysis method. King (1998)'s temp late analysis procedure was adopted in the study, which could be divided into the steps listed as follows. 1) developing template. The study took four stages of Gibson's (1995) parent empowerment as the topics in the consultation of the consultees, including finding the reality, considering setback, criticizing and introspecting, and controlling. The maintaining was used as the core of their resilience cultivation and development after consultation and used in the coding of the tracking interview and questionnaire. 2) revising template. The initial temp late was revised in four steps, including inserting codes, deleting codes, extending the coding range and changing the classification in high layer. 3) confirming template. The codes of the text of the consultation process were classified based on the top 5 topics of the empowerment coding system in the confirmed template, including "Exploring and Tracing Back to the Origin", "Initiating Fortune from the Extreme Adversity", "Flourishing Strengths", "Comprehending from Converting Thoughts", and "Facilitating from Being Proficient". During the post interview and tracking questionnaire, the development of consultees' resilience was analyzed and classified, and the topic of resilience development was named as "Diversifying Crises into Safeties". 4) explaining template. The study explained the theme and elements of the confirmed template, as well as the empowerment process.

\section{Results}

\subsection{Development and Connotation of the Consultation Empowerment Template}

The "finding reality" in the initial template is changed into "Exploring and Tracing Back to the Origin" by the researcher, to explore the context and nature of physical and mental problems of children. Tracing back to the origin can help consultees find the self-significance, further drive the review on the emotion, cognition and behavior, and enhance the diverse development of parenting experience. Gibson (1995) emphasized in his study that the setback has the potential to be catalyzed. However, when it comes to the Chinese cultural context, the 
natural transition between bad and good fortune is emphasized. Therefore, "Initiating Fortune from the Extreme Adversity" is used to present the demand of changing inner pain to joy for the objects in the study. During the transition, an expectation of changing is derived, which will become the driving force of pursuing dynamic balance.

"Flourishing Strengths" is a new topic proposed by the study, which means collecting the current positive experience, atmosphere and emotion in heart, changing them into the advantages of changing positively, and using proper strategies to meet the challenges in current and future contexts to get positive effects. According to the meaning of the topic, the successful strategies in the positive experience can be used to reconstruct the advantages; the positive atmosphere can enhance the cognition towards positive characters and deepen the positive transformation of emotions; and the positive emotion can release pressure by resilience effect.

The "criticizing and introspecting" in the initial template is revised into "Comprehending from Converting Thoughts" to highlight the cognitive transition and digestion presented by the consultees in the study which help them break through difficulties. The "controlling" in the confirmed temp late is changed into "Facilitating from Being Proficient". The first connotation shows the consultees can listen to the heart from the external authority, which presents the self-dependence. The second connotation shows the consultees can adapt themselves to the parenting role, which forms the external behaviors of parenting self-dependence and the inner experience of parenting nourishing to accumulate the parenting resources and energy.

The "maintaining" in the initial template is similar to development of a kind of resilience ability, which extends the spatiality and timeliness of the empowerment. The resilience ability in the study is defined as a healthy adaption process produced internally to get the positive result when individual is facing crises or difficulties. It is a context and ability of "Diversifying Crises into Safeties". Therefore, "Diversifying Crises into Safeties" is used to show the development of resilience ability of the consultees after consultation. The "Diversifying Crises into Safeties" contains the meanings of context and adaption. The consultation effect perceived subjectively can be transformed into a kind of adaption energy, providing consultees with the changing expectation and the energy of meeting the future challenges. That meaning is named adaption energy. Moreover, with the sufficient energy, the ability improvement acquired from consultation by consultees will be transformed into the presentation of adaption strategy, which will give full play to the parenting efficacy and help consultees adapt themselves to the possible crises and challenges freely. That is the ability meaning of "Diversifying Crises into Safeties", named as adaption ability. 


\subsection{Connotation Analysis on the Empowerment Effects}

Then the study is then going to analyze the connotation of the empowerment effects to find the satisfaction level of the consultees to the current consultation, and to enhance their firmness of the future parenting commitment and the possibility of parenting practices by improving the adaption ability. The analysis is explained in two parts.

\subsubsection{Adaption Energy}

The most important consultation effect felt by consultees is the "parenting mutual assistance", which means the consultees and their children get help from others to improve practically. This connotation focuses on deepening atmosphere nourishing, and places the parenting practices in a context of systematic cooperation rather than only consultees themselves. Moreover, "children development" is another important consultation effect, which indicates by the increase of consultees' knowledge and skills, the consultation model is indeed helpful to the positive adaption of children and shows the improvement of the physical and mental adaption of children. In addition, "relationship improvement" refers to the relationship improvement between the consultees and their children and family members, which is also an important effect of the consultation model. When extending the family to the system, consultees can feel the support and assistance from the system. Therefore, the consultees can be benefited a lot from the mutual assistance and cooperation in the system.

\subsubsection{Adaption Ability}

The adaption ability developed by the consultees is mainly derived from "parenting ability", "cognition transition", "self-nourishing" and "resource application". The study aims to develop the empowerment model of the consultees. The greatest improvement of the consultees is getting parenting knowledge and skills subjectively from consultation, with the percentage of $31 \%$ shown in Figure 5. Moreover, the "cognition transformation" is another important adaption advantage for consultees, with the percentage of $28 \%$. The cognitive

adjustment ability depends on whether the consultees can build proper cognition and expectation based on the different physical and mental conditions and developing stages of the children. 


\subsection{Construction of the Empowerment Model of consultees}

According to the findings above, the empowerment connotations and evolution process of consultees are drawn in Figure 1.

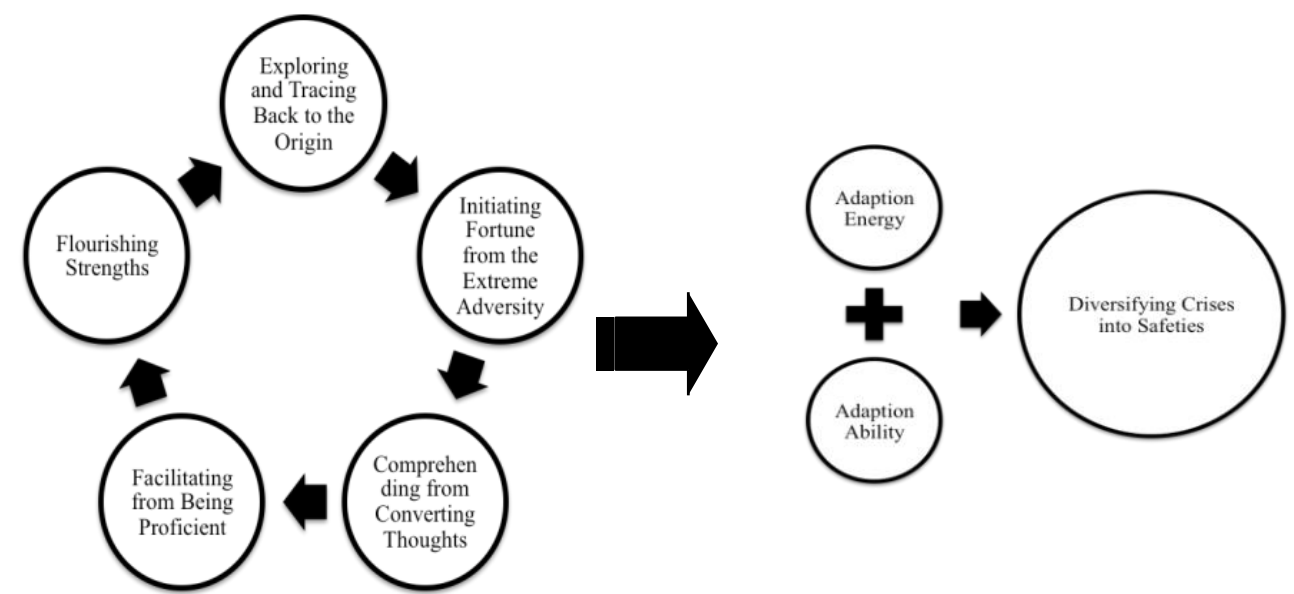

Figure 1. Conceptual map of the empowerment connotations and evolution process of consultees

The consultation process contains five empowerment topics. According to the evolution process concluded by the researcher, the topics are ranked as "Exploring and Tracing Back to the Origin", "Initiating Fortune from the Extreme Adversity", "Comprehending from Converting Thoughts", "Facilitating from Being Proficient", and "Flourishing Strengths". Although ranked based on the important stages that topics appear, the five topics actually appear in the whole consultation process, and develop alternatively based on the physical and mental situation and the context change, which will drive the development of empowerment.

\section{Discussion}

The empowerment transformation takes "Exploring and Tracing Back to the Origin" and "Initiating Fortune from the Extreme Adversity" as the principal line to further form the power of exploring the essence and changing pain to joy. During the process of empowerment transition, consultees tend to explore the inspiration of their situation by observing and introspecting. Therefore, by means of the psychological digestion, they turn to introspect the imbalance of the relationship in the difficult situation, form a circulation of exploring, understanding and transferring in the empowerment transformation process. It also reflects 
Chinese people always break through the external difficulties by introspection, and can form a power of changing pain to joy internally (Lv Zheng-da, 1987; Hsu, 1953).

When consultees realize the dynamic nature of life and learn to accept the situation and create another possibility, their mind will be changed. When they change their mind, the feedbacks in the practices will become positive, and their self-dependence will be enhanced. Shown in the consultation, the self-dependence means consultees can talk with the consultants equally. The consultees' self-dependence should be tolerant, and the positive parenting feelings should be formed by developing self-dependence strategy and implementing the positive experience. Finally, the advantages of atmosphere, experience and character are collected as the advantage foundation of the subsequent adaptation power.

The result of empowerment is to build parenting wellbeing by extending the relationship nourishing, stimulate the parenting adaption by introspection, and further develop the ability of diversifying crises into safeties. In the consultation benefits perceived by the consultees, "parenting mutual assistance" and "children development" are two important connotations, which describe the consultees feel the supporting atmosphere of systematically self-assisting and assisting children from the improvement of parent-children relationship or family relationship, and show a kind of wellbeing based on relationship. The important connotations of the adaption development are "parenting ability" and "cognition transition". Therefore, consultees in the study identify the proper position by internal cognition transformation, to keep the power of adjustment for the future parent-child context and events. The presentation of the empowerment result shows the connotation of the core of resilience, namely, assisting the difficulty of self-transition by relationship nourishing and support power, and also shows the preventative function of the consultation is achieved (Dougherty, 1995; Lin Han-yun, 2011; Qiu Xian-hui, 2003).

According to the results of empowerment, the connotation of empowerment model echoes with the binary harmony, relation harmony and social orientation established by the researcher, which can lead consultees to find a nourishing parent-child and family relationship to develop the self and parenting roles harmoniously.

As for the study limitation, the formal study only adopts four female parents to construct the empowerment model. Moreover, all the objects are female which can't cover the consultation connotation and the change of the model caused by gender. There is no domestic study on the development of the parent consultation model from the perspective of positive psychology, and the study is only a preliminary exploration. Due to the object limitation, the study only presents the empowerment development of the consultees by temp late analysis. Therefore, the consultation effects are based on the subjective perception of the consultees and 
the explanation of the researcher. Besides, the effects of the model in the study are not shown in the data that is resulted from strict experimental design without any interference factor, which limits the generality of the effects of the model.

The further studies can be designed to discuss the obstacles and stimulating factors among different groups classified according to the consultation engagement level, and construct a more complete consultation process to help consultants develop more complete expertise of consultation. Different temp late studying methods can be adopted to obtain more viewpoints. As for the discussion of the consultation effects, the changing trend can not only be presented by quantified data, also the changing context can be interpreted by other qualitative ways. For example, the changing model can be constructed by the grounded theory, and the social connotation and advocated ideas of the changing context can be proposed by the critical hermeneutics.

\section{Acknowledgements}

The author(s) declare that there is no conflict of interest.

\section{References}

Chin, J. L., Liem, J. H., Ham, M. D., \& Hong, G. K. (1993). Transference and empathy in Asia American psychotherapy: Cultural values and treatment needs. Westport, CT: Praeger.

Dougherty, A. M. (1995). Consultation: Practice and Perspective. Pacific Grove, California: Books/Cole Publishing Company.

Gibson, C. H. (1995). The process of empowerment in mothers of chronically ill children. Journal of Advanced Nursing, 21, 1201-1210. https://doi.org/10.1046/j.13652648.1995.21061201.x

Goldenberg, H., \& Goldenberg, I. (2008). Family therapy: An overview (7th ed.). Belmont, CA: Thomson.

Guo, N. W. (2011). Combination of Life Education and Positive Psychology: Positive Psychology Derived from Neuropsychology. Speech conducted on the "Life Education \& Positive Psychology Seminar" of the Department of Clinical Psychology, Fu Jen Catholic University.

Holcomb-McCoy, C., \& Bryan, J. (2010). Advocacy and empowerment in parent consultation: Implications for theory and practice. Journal of Counseling \& Development, 88, 259268. https://doi.org/10.1002/j.1556-6678.2010.tb00021.x

Lin, H. Y. (2011). Start-up Resilience: Narrating Consultation with the Perspective of Resilience. Counseling \& Guidance, 304, 44-47.

Lin, M, Z, (2002). Consultation, Indispensable Part in Primary School Tutoring. Guidance Quarterly, 38(2), 8- 16.

Lin, R. J. (2007). The Action Research of Teacher Consultation in the elementary School: A School Counselor's Practical Story. Doctoral Dissertation of Educational Psychology $\&$ Counseling Institute, National Taiwan Normal University.

Qiu, X. H. (2003). The Basic Concept of Consultation. Counseling \& Guidance, 216, 2-10 
Qiu, X. H., \& Wu, P. L. (2004). The Effects of a Parent-Adolescent Communication Consultation Group. Bulletin of Educational Psychology, 36(1), 35-57.

Wang, L. F. (2002). Study on the Connotation of Dealing with Primary School Cases: Practical Experiences and Future Orientation. Taipei: Pro-Ed Publishing Company 